\title{
Flexible Power Converters for the Fault Tolerant Operation of Micro-Grids.
}

\author{
F. Genduso, R. Miceli, member IEEE, G. Ricco Galluzzo
}

\begin{abstract}
The progressive penetration level of Distributed Generation (DG) is destined to cause deep changes in the existing distribution networks no longer considered as passive terminations of the whole electrical system. A possible solution is the realization of small networks, namely the Micro-Grids, reproducing in themselves the structure of the main production and distribution of the electrical energy system.

In order to gain an adequate reliability level of the microgrids the individuation and the management of the faults with the goal of maintaining the micro-grid operation (fault tolerant operation) is quite important.

In the present paper flexible power converters and a companion control algorithm for the fault tolerant operation of microgrids are presented. The effectiveness of such an algorithm and of the fault tolerant power converters are verified through computer simulations.
\end{abstract}

Index Terms-Micro-Grid, flexible power converters, Fault Tolerance, Faults.

\section{INTRODUCTION.}

The forthcoming penetration of the Distributed Generation (DG) is destined to cause a deep change in the structure of existing distribution networks and also in the future planned ones. Actual networks, in fact, have a radial topology and are passively managed with the only goal to feed the end users, but they are also destined to reach an active role via implementation of the typical functions of load management, demand side management, generation curtailment.

A solution prospected to optimize the benefits and to minimize the inconveniences due to the DG, is the realization of small networks (namely the micro-grids) reproducing in themselves the structure of the power generation and distribution system at a smaller scale. It is possible, for the isolation or the connection of the micro-grid to the main distribution network, to put the difference between autonomous (stand alone) and interconnected micro-grids; in this last case the micro-grid may be assimilated to active network cells constituted by a set of electrical loads and generators all connected to the main network through a single interfacing node.

The revolutionary profiling scenarios do not consider the distribution system as a passive termination of the larger scale system, but as a network able to guarantee a "connectivity", i.e. the connection between producers and end users satisfying these last ones in terms of economy, availability affordability and reliability, with economical benefits coming out from the electrical market liberalization.

F. Genduso, R. Miceli, and G. Ricco Galluzzo are with the DIEET - Dipartimento di Ingegneria Elettrica, Elettronica e delle Telecomunicazioni Università degli Studi di Palermo, email: genduso @ dieet.unipa.it, miceli@dieet.unipa.it, giuseppe.ricco@unipa.it
Also actually, the distribution systems are the subject of a very profound revision process with the goal of a deeper implementation of automation functions in order to increase the power quality and the services. The application to these system of an high level of information and communication technologies (ICT) will track the road map for the diffusion of the micro-grids that, still nowadays, are under investigation both on national and international contest.

All the hypothetical scenarios imply transformation and development processes that include several issues concerning technical aspects ratios interesting for the next studies and researches.

To get the target of a wider dissemination of DG it will be necessary to overcome the manifold of problematics issued by the network management no longer characterized by unidirectional power flows, as for example, those regarding the non optimal development of lines and transformers, the complexity in the voltage regulation the unwanted islanded operations and the increased complication in the protection issues, the development of fault tolerant operation systems. [1], [2]

In this paper the possibility of a fault tolerant operation of the micro-grid is investigated. Fault tolerant mode has a capital importance in the management policy of the microgrid within a free market; in fact it implies consequences in terms of the continuity and power quality, with particular reference to the autonomous micro-grid, characterized by reliability levels smaller than those ones interconnected to the main distribution system. For the fault tolerant operation of micro-grids a particular approach to the diagnosis of fault for the various component of the micro-grid is followed and by the determination of the most proper fault indexes fault tolerant control strategies are to be employed. For this reason flexible power converters usage is of a capital importance. Fault tolerant operation of the electronic power converter have been studied for years in view of the application to electrical drives. In the case of this paper the fault tolerant operation of power converters is considered in order to ensure an increased degree of power quality and availability of the generated electrical energy.

\section{DiAgnosis SYSTEM, MONITORING AND FAULT INDEXES.}

Flexible power converters for the fault tolerant operation of a micro-grid requires a proper Diagnosis System to be developed with. The ideal diagnosis system for the fault detection in an electrical system (electrical machines, power converters, electrical networks) has to be able to give a secure 
indication of anomaly not only of a fault presence but also of its typology and, eventually, to give also useful information on its location, entity and on its dynamical evolution during time.

Even if the modes of the fault appearance depends on the devices and on the operational environment, it is however possible to detect some effects or phenomena in order to define the fault indexes. With the aim to realize an effective and economic diagnostic system all the fault and incipient fault indicators (or indexes) are to be monitored.

In order to individuate all those indexes, it is, first of all, necessary the verification through physical observations of the system states and of the quantities to be investigated undergoing through modification in the case of a fault or a prefault condition. After this, the presence of a deterministic or not bound between the monitored quantities and the fault condition is needed to extract a numerical model in order to make a comparison between the different states of the systems.

When the numerical model is really available all the comparison may be previously verified through simulation instruments.

As for the state of the art the effect of fault may be investigated in the frequency domain, it is necessary that each single signal acquired in the time domains if fastly converted in the frequency domain with the Fourier Transform and for matter of best performances the FFT algorithm is very often used. From the frequency spectrum, in fact, it is sometimes possible to get the informations about the hypothesized fault or prefault condition.

\section{FAULTS AND FAULT IN FLEXIBLE CONVERTERS FOR MICRO-GRIDS}

Due to the frequency and voltage variation in the DG systems, the use of electronic power converters is mandatory to ensure the specifications that make the generated energy suitable to be injected into the network. In the field of DG ancillary systems are also introduced. Among these the synchronization to the main network is on of the most important. This exigence is really of a capital importance for all the micro-grids for which the islanding operation is taken into account; in this case, in fact, a fault occurrence requires the disconnection from the main network in order to avoid the propagation of the fault to the public utilities, and the fast reconnection to this when the fault condition is eliminated.

The reliability of the modern power converters has limited the probability of faults occurrence, even more for those localized on the single power devices. Actually the most frequent faults are represented by short circuit and permanent switching off of a single device. Among these two faults the second appears more often. On the other side, electronic power devices are protected by series connected fast fuses so that the short circuit condition fastly evolves to the open line condition [5]. During the last years the construction of power converter is more often addressed to the usage of Power Electronics Building Block units (PEBB), i.e. circuit cells with various connected components and integrating protection inside conceived to realize the most diffused power convert

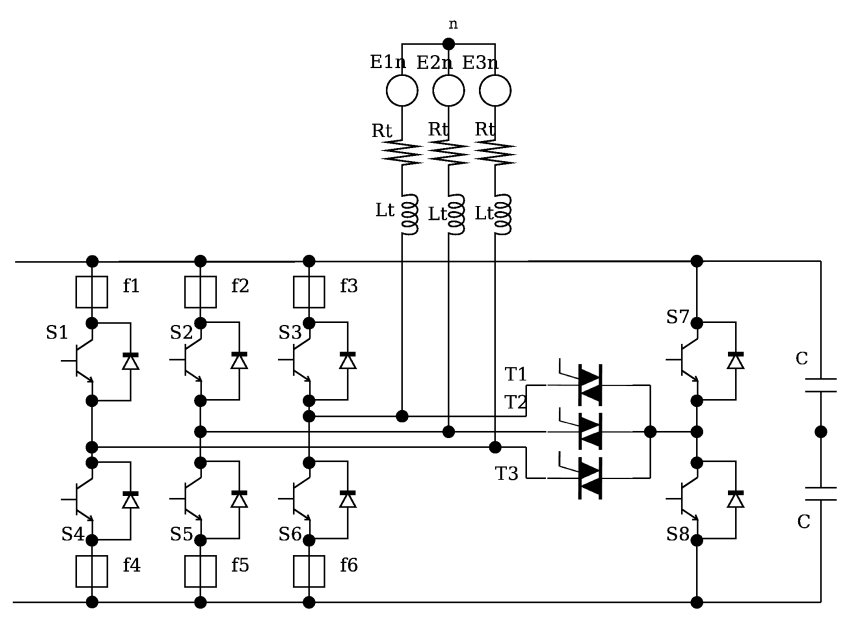

Fig. 1. Topology of redundant fault tolerant converter

topologies with a high degree of modularization [3], [4], [5]. For distribution systems it has been demonstrated that PEBBs can be used to develop diagnostic and protection tasks thanks to their integrated sensors with the advantage of the elimination of external sensors and external protection devices for the silicon components [4], [5].

Anther interesting aspect issued to PEBBs is their ability to realize power conversion system that are also "fault tolerant" i.e. able to continue their operation after a fault occurrence with acceptable condition for the end user. This means in practice "with a low degree of power quality decrease". Furthermore there are some cases in which a significant decrease of power quality is only transient with a very small duration. Taking the intervention modes and the converter topologies into account, the fault tolerant converter may be divided into two big families:

- the redundant converters

- the non redundant converters.

The first ones are characterized by the presence of some additional not switching devices during normal operation and used as back-up units in the case of a fault occurrence with the loss of a converter leg. The ratings of the additional devices are practically the same of the main devices because they must be able to ensure the "normal operation" of the converter with the same voltage and current values. Figure 1 shows the case of a redundant fault tolerant converter. As a matter of fact this converter has an additional leg connected to each of the three phases of the load with a static device able to bidirectional conduction of current. The static version of the breaking device is preferred to the mechanical one because the switching on is to be made at load condition, with minor hazards and risks and faster than with mechanical breakers. The fault diagnosis and the fault tolerant operation of the redundant converter is articulated as follows:

1) the individuation o the faulted leg;

2) the disablement of the faulted leg drivers;

3) the gating of the triac connected to the faulted leg; 


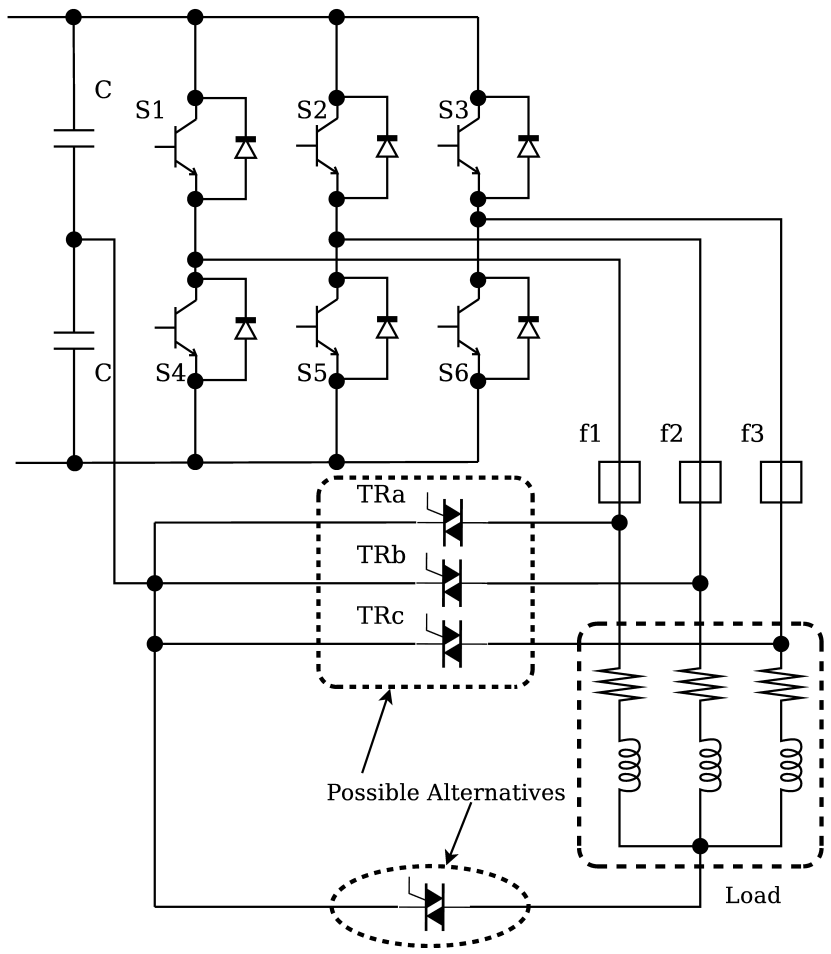

Fig. 2. Topology of non redundant Fault tolerant converter

4) the transfer of the switching control to the redundant devices.

The speed intervention is high enough to not determine a significant degradation of the power quality except for few commutation cycles or at least for a single cycle with a more sophisticated control. In an enhanced version of this converter more back up devices are present (making more legs available) and obviously a greater number of triacs for the connection to the load is needed.

Figure 2 shows a non redundant fault tolerant converter: in this case the additional components are only the triacs connecting each phase of the load, after the protecting fuses, at the middle point of the DC Link. The switching of such a triac guarantees the enclosure of the current path both in the case of the fault of a silicon device and in the case of a phase interruption before the load. The ratings of the triacs have to be chosen in order to enable the phase current but with a reduced voltage in the linear range. it can be noted that the non redundant converter topology is similar to that of a B4 inverter with DC link capacitor splitting. However the B4 inverter is not intended to be a fault tolerant converter but in order to realize a cheaper one with a reduced equipment.[6]

In the case of star connected loads with accessible neutral point only this point will be connected to the DC Link middle through the triac [7]. This happens when the current loss on the faulted leg is considered acceptable for the exercise of the machine connected to the converter.

For non redundant converters the fault tolerant operation is articulated as follows:

1) the individuation of the faulted leg;
2) the disabling of the drivers of the faulted leg;

3 ) the switching on of the triac connected to the faulted leg;

4) the modification of the converter's control strategy (modification of the pulses sequence).

The fault on a single device reduces of two units the number of the realizable conduction states, but in spite of this it is still possible to follow the voltage reference with a suitable precision even if the normal linear range of the converters is reduced. In the case when the voltage asymmetry margins are narrow the usage of passive symmetrizing cells must be taken into account too.

\section{DIAGNOSIS OF THE ELECTRICAL AND MECHANICAL FAULTS ON ROTATING MACHINERY FOR THEIR FAULT TOLERANT OPERATION.}

In the micro grid environment, besides the usage of fault tolerant converters there is the need to diagnose the generators developing a coordinate strategy for intervention in order to ensure the effective fault tolerance of the whole system as possible.

In fact, the modern electrical machines, power converter ad the generation systems need a pointed-up identification of their operating condition with proper monitoring systems, sometimes also in spite of their high cost. In particular the recognition and the identification of the fault condition allow the operators to take specific procedures of planning only limited machine stops with the aim to allow them to continue their operation in some tolerable prefault conditions and in accordance within the planned intervention times.

In this way the times of service interruption are sensibly reduced and the planned maintaining and repairing may be conduced in a simpler way because the prefault condition is suddenly recognized avoiding it evolution to disruptive fault. In order to implement the correct operational strategies it is mandatory for the conversion system to include proper diagnostic services to individuate and localize the fault condition, insulating so only the malfunctioning apparatuses and devices.

As known, the most part of faults in electrical machines, both in generating and motoring operation, is localized in the stator and rotor windings and on the bearings for the mechanical part. the electrical fault are essentially due to insulation faults and generally tends to evolve from the small short circuit within a small number of turns [9]. The bearings faults amounts typically the rate of $50 \%$ of all the faults in general and are due to mechanical degradation caused by the relative motion between the fixed and the moving parts of the machine [10].

In the technical literature a large number of diagnosis method aimed to the individuation of the mentioned fault types were proposed. The best known are the following:

1) the method of partial discharge, suitable for machines with rating voltages higher than $4 \mathrm{kV}$;

2) the method based on the measurement of the axial flux component, suitable for low voltage machines;

3) the method based on the measurement of the inverse component sequence due to the presence of phase asymmetry under a fault condition; 
4) the method based on the measurement of stator vibration useful in the case of both electrical and mechanical fault conditions;

5) the method based on the individuation of harmonic components on stator voltages and currents useful for the determination of both electrical and mechanical fault conditions.[9], [11], [12][13][14], [15]

Each of the diagnosis method has its application field and a particular range for which it can guarantees the best results, for example the partial discharge method gives significant results id used in the case of electrical generators with rating voltage higher than $4 \mathrm{kV}$. Its natural application is in the field of high power Wind Generators. Instead the method based on inverse sequence component does not suffer for this limitations. On the other side the method based on the presence of stator vibrations requires the execution of several test in which the measurements needs a lot of mathematical manipulations (spectral analysis via FFT or similar algorithms) and also require additional equipments for the revelation of the mechanical vibration such as accelerometers or different motion sensors mounted on the machine casing, or on the shield when the axial vibrational components are of interest.

The choice of diagnosis method is a critical point in planning the electrical systems and require the widest experience in the conduction of electric plants tanking the novelty of DG into account. Also economical issues are very important in the organization of a fault tolerant micro-grid whole project.

\section{ARCHITECTURE OF A DIAGNOSIS ALGORITHM FOR A FAULT TOLERANT MICRO-GRID}

The identification of such a fault, require the execution of several measurements, or better, a continuous monitoring of the interesting quantities. The values of such quantities are to be compared with the faults indexes whose determination starts from experimental investigation or by simulation results based on the mathematical model of the system under fault condition.

The fault detection through comparison between faulted and unfaulted conditions require a preliminary phase of intensive studies for the realization of comparison tables used in dependency of the considered micro-grid structure and on the interconnection of it with the main network. For this determination several "ad hoc" created simulation software are also available allowing the simulation of the behavior of each single device in order to establish the most suitable fault indexes [16].

The mentioned comparison tables can be easily implemented on digital architectures and "hardware in the loop" based on FPGA (Field Programmable Gate Arrays). The employment of such a system presents some advantages respect to the ASIC (Application Specific Integrated Circuits) Architecture [8].

However it must be observed that in some cases the fault tolerant operation is not possible at all, or become more problematic as in the cases of multiple faults, or when a novel abnormal condition is not recognized by the fault model.

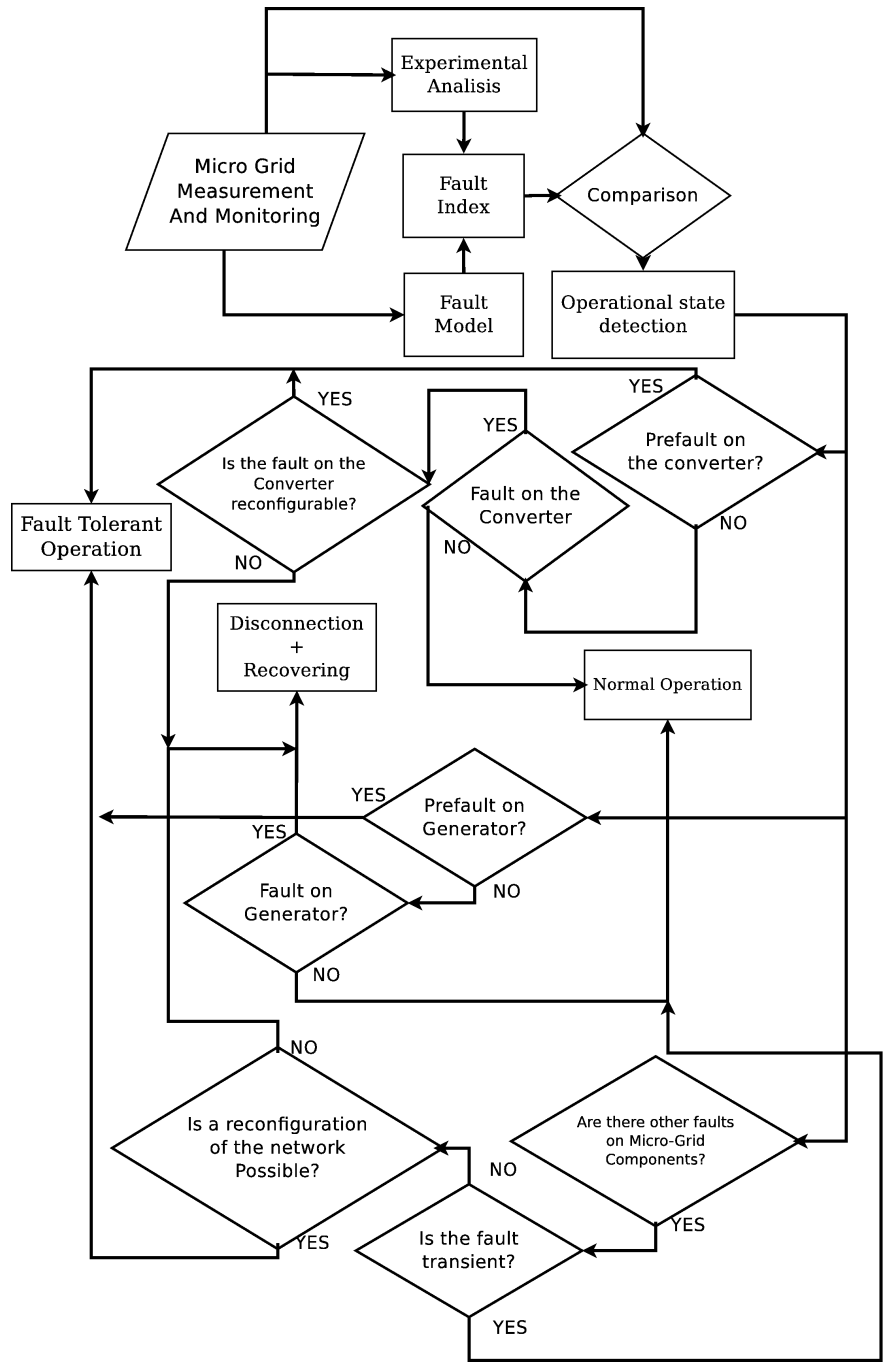

Fig. 3. Flow chart of the proposed approach

In this section the Authors propose the structure of a diagnosis algorithm for the fault tolerant operation of a microgrid. This one consider also the disconnection when the system recovery is not possible, but reduces the cases in which disconnection is used. This algorithm is illustrated with the flow chart of figure 3 . The easier way to follow it is trough to separate the ways in which it acts for different class of apparatuses (power converters, generators, other electrical machinery and lines).

In particular, for the converters the following possibilities are contemplated:

1) in the case of a prefault the converter operate in fault tolerant mode, warning about its state;

2) if the fault contemplates the possibility of a reconfiguration of the converter it operates in fault tolerant mode;

3 ) if a fault with no possibility of reconfiguration is detected, the same converter is disconnected from the grid and turned off waiting for the repairing made easier by the knowledge of the fault type.

As for generators and other electrical machines the following possibility are contemplated: 
1) if a prefault condition, tolerable until the time of intervention on the system, is detected the generator continues with fault tolerant operation mode; the warning about the prefault condition allows, in each case, the setting up of the various procedures needed by the recovery without the disconnection of the generator from the main;

2) if a fault condition is revealed the generator have to be disconnected for the necessary recovery and repairing operations.

For all the other components of the network the following possibilities exist:

1) if the fault has a transient nature, it can be recovered, simply by the process of disconnection and reconnection of the faulted brunch or equipment. After this the microgrid restarts its normal operation.

2) if the fault has a permanent nature and it results possible to reconfigure the micro-grid this reconfiguration is mandatory to reach the fault tolerant operation.

3 ) if the fault has a permanent nature and the micro-grid is not reconfigurable, it must be entirely disconnected by the main network (if previously connected), deactivated and recovered.

For all that concerns the faults on the distribution lines on distribution, it must be remarked that the entire DG must be disconnected without any doubt with its interface devices in order to ensures the correctness of the procedure for fault elimination by disconnection and reconnection [2].

In fact in case of a poliphase fault the presence of DG, apart the passive loads, the devices for the selection of faulted branch and the eventual remote controlled devices could not sense correctly the fault condition. In the case of procedure implemented with device for the selection of faulted brunch it starts only if the fast reconnection of the breaker fails and the further actions are to be made in strong coordination with the line breaker operation trough local voltage measurements really discriminant for the recognition of the fault condition. If the DG would continue to work during the absence of network interconnection there would manifest strong anomalies caused by the fault enforcement by the generators.

\section{Simulation OF THE DiagnOSIS SYSTEM INTERVENTION: FAULT TOLERANT OPERATION OF THE CONVERTER}

In order to verify the validity of the proposed diagnosis system several numerical simulation have been made with the help of the MATLAB - SIMULINK software package. The simulation simulations required the modeling of the single components of a micro-grids, of the control system and the implementation of the diagnosis system through the flow chart of Fig. 3.

The case study hereafter presented verify through simulation of the diagnosis system intervention in the specific case of a fault on the power converter. This situation has been chosen in virtue of its complexity because power converter need a deeper degree of reconfiguration, passing also through the modification of the control strategy. Simulation results are illustrated in figs. 4 to 6 showing how the system is able to detect the fault condition and to communicate with the control system modifying the control strategy of the converter in order to guarantee the minimal degree of power quality degradation. Fig. 4 shows the the converter phase voltage both at unfaulted (upper figure) and faulted leg (lower figure) after the faults occurred.

The voltage waveforms will be quite different from those with unfaulted converter condition and the faulted leg voltage exhibit the greatest difference. Furthermore the converter linear range is in each case restricted. Linear range is quite important in order to ensure the minimal asymmetry for the current system. An over-modulation running requires a modification of the displacement angle between the reference voltage in order to gain a sub optimal symmetry condition.

Fig. 5 shows converter's phase currents after fault occurrence.

A symmetrical current system is gained using two different reference voltages for unfaulted legs, with a phase displacement of about $60^{\circ}$ degrees. This displacement ensure the minimal asymmetry of the current system.

Finally fig. shows the voltage imbalance on the two DC Link capacitors.

Considering the non redundant converter topology it is interesting to note that as the faulted phase terminal is connected to the DC Link middle point the phase current is injected into the DC Link itself. This causes obviously a fluctuating difference in the capacitor voltages. However, this difference does not affect significantly the entire value of the DC Link voltage.

\section{CONCLUSIONS}

In this paper, after the presentation of the main aims of the diagnosis systems and of the fault tolerant converters, the main diagnosis techniques for the electrical machines were recalled and finally a novel approach for the fault tolerant operation of the entire micro-grid was presented and proposed.

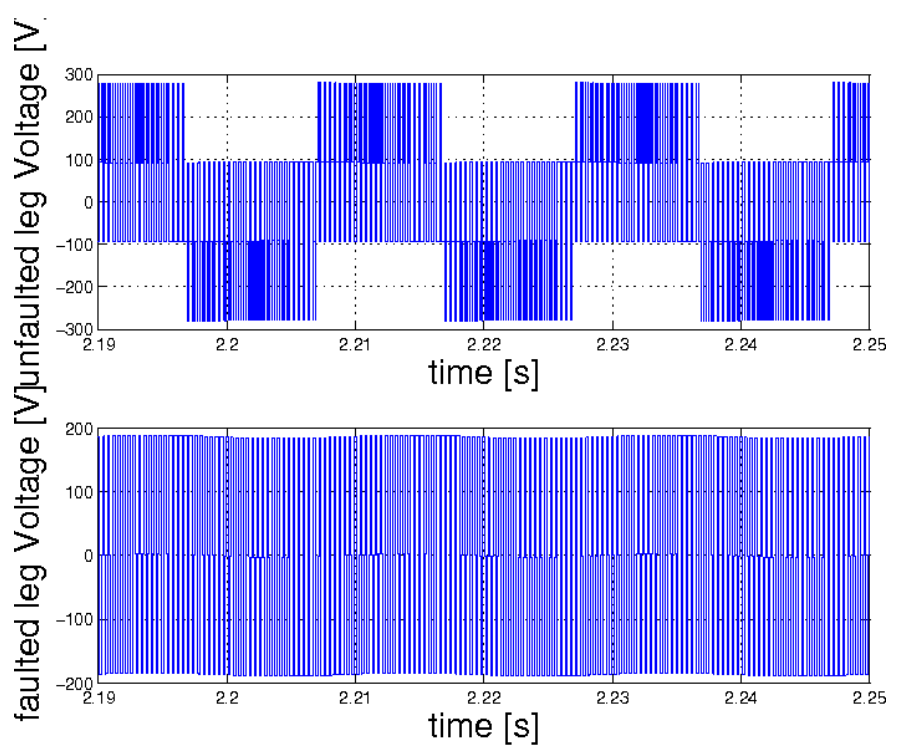

Fig. 4. Phase voltage at unfaulted and faulted converter leg 


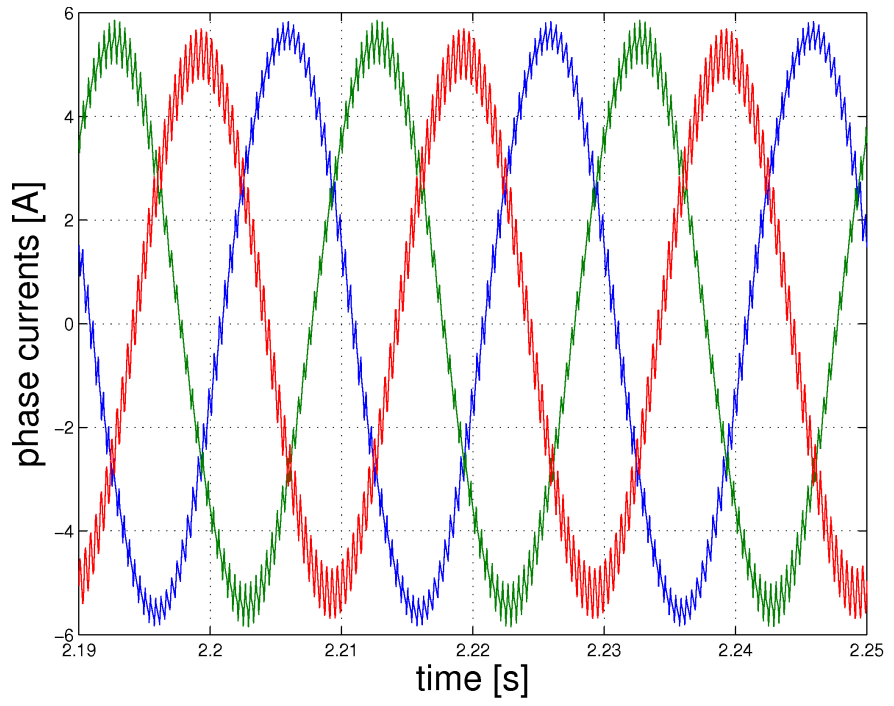

Fig. 5. Converter currents after fault occurrence

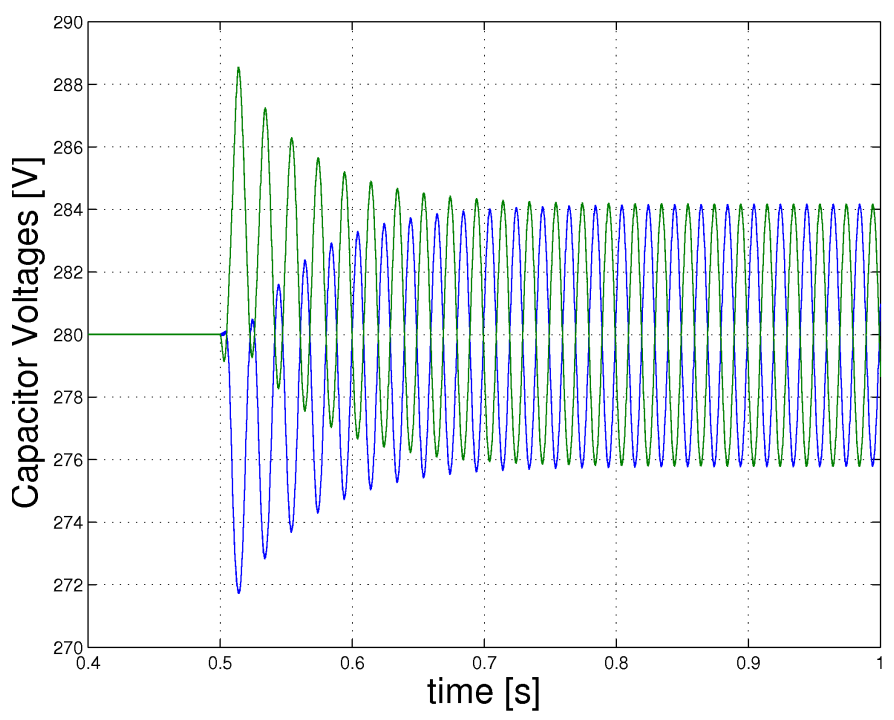

Fig. 6. Voltage imbalance through DC Link Capacitors

The development and the adoption of this system is essential both for the optimal development of electrical power produced by renewable sources characterized by an high degree of randomness and for the assurance of high level of reliability and power quality, both of capital importance for all the end users.

The architecture proposed for such diagnosis systems is suitable for the used in conjunction with intelligent algorithms for fault detection and result to be simple to implement on low cost and user friendly hardware ad, e.g. FPGAs.

Computer simulations presented in the paper show that the proposed system is able to recognize the fault conditions (in particular in the delicate case of power converters) and also is able to reconfigure the converter control assuring a minimal degree of power quality degradation.

\section{ACKNOWLEGMENTS}

This work was financially supported by MIUR - Ministero dell'Istruzione dell'Università e della Ricerca (Italian Ministry of Education, University and Research), PRIN 2008, and by SDESLab (Sustainable Development and Energy Saving Laboratory) of the University of Palermo and was partially supported by the project BeyWatch IST-223888 funded by the European Community, web page: http://www.beywatch.eu/

\section{REFERENCES}

[1] D. Castelli, B. Ceresoli, A. Cerretti, E. De Berardinis, G. Simioli, E. Tironi, F. Vertemati, "Integrazione e gestione della generazione distribuita collegata alla rete di distribuzione MT/BT", workshop AEI - Specialistic group on pubblic utiltiy on Distributetd Generation: motivations and perspectives, Milan May 82003.

[2] A. Cerretti, S. Botton, S. Sartore, "I problemi del gestore della rete di distribuzione", AEIT, Seminario didattico - Medium and small generation: interaction with network and market, Oct 22-23 2007 - Rome Italy.

[3] J. C. Piff "Power Electronics Building Blocks program: PEBB bringing a whole new perspective to power control and distribution - PEBB Best Business Practices", 23 Jun, 2009, http://www.findarticles.com.

[4] M. Baran, N. R. Mahajan, and A. W. Kelley, "Use of PEBB Converters as Current Limiting Circuit Breakers", submitted to IEEE Transactions on PELS, Aug 2004.

[5] J. C. Salmon, "Current overload protection features of hybrid inverter drives", Proceedings of the 1992 International Conference on Power Electronics and Motion Control, 1992.

[6] Frede Blaabjerg, Sigurdur Freysson, Hans-Henrik Hansen, and S. Hansen "A New Optimized Space-Vector Modulation Strategy for a Component-Minimized Voltage Source Inverter" IEEE TRANSACTIONS ON POWER ELECTRONICS, VOL. 12, NO. 4, JULY 1997

[7] B. A. Welchko, T. A. Lipo, Thomas M. Jahns, Steven E. Schulz, "Fault Tolerant Three-Phase AC Motor Drive Topologies: A Comparison of Features,cost and limitation", IEEE Transactions on Power Electronics, Vol. 19, No. 4, July 2004

[8] S. Karimi, A. Gaillard, P. Poure, S. Saadate, "FPGA-Based RealTime Power Converter Failure Diagnosis for Wind Energy Conversion Systems", IEEE Transactions on Industrial Electronics, Vol. 55, No. 12, December 2008

[9] H. Behbabanifard, H. Karshenas, A. Sadoughi, "Non-Invasive Online Detection of Winding Faults in Induction Motors - A Review", 2008 International Conference on Condition Monitoring and Diagnosis, Beijing, Chine, April 21-24, 2008.

[10] Wei Zhou, T. G. Habetler, R. G. Harley, "Bearing Condition Monitoring Methods for Electric Machines: a General Review", IEEE International Symposium on Diagnostics for Electric Machines, Power Electronics and Drives. SDEMPED 2007, 6-8 Sept. 2007.

[11] G. Stone, J. Kapler, "Stator Winding Monitoring", IEEE Ind. Appl. Mag., Vol 4, Nr. 5, pp. 15-20, Sept./Oct. 1998.

[12] J. Pennman, H. G. Sedding, B. A. Lloyd, W. T. Fink, "Detection and Location of Interturn Short Circuits in the Stator Windings of Operating Motors", IEEE Trans. Energy Convertion, Vol. 9, pp. 652-658, Dec. 1994.

[13] H. A. Tolyat, T. A. Lipo, "Transient Analisys of Cage Induction Machines under Stator, Rotor Bar and End Ring Faults", IEEE Trans. Energy Convertion, Vol. 10, Nr. 2, pp. 241-247, June 1995.

[14] N. Arthur, J. Pennman, "Induction Machines Condition Monitoring with Higher Order Spectra”, IEEE Trans. Ind. Electron., Vol. 47, Nr. 5, Oct. 2000.

[15] C. Cavallaro, A. O. Di Tommaso, R. Miceli, A. Raciti, G. Ricco Galluzzo, M. Trapanese, "Monitoring and Diagnoses by a Vibration Analysis Procedure of Incipient Failures in Squirrel Cage Induction Motors Due to Cracked or Broken Bars", SPEEDAM 2004, Capri (Italy), June 16th to 18 th, 2004, pp. 839-845.

[16] W. Feero, D. C. Dowson, J. Stevens, "White Paper on Protection Issues of the Micro-Grid Concept", Consortium for Electric Reliability Technology Solutions, March 2002, Albuquerque, NM. 